\title{
Mechanistic Implications for the Chorismatase FkbO Based on the Crystal Structure
}

\author{
Puneet Juneja $^{1, \dagger}$, Florian Hubrich ${ }^{2, \dagger}$, Kay Diederichs ${ }^{1}$, \\ Wolfram Welte ${ }^{1}$ and Jennifer N. Andexer ${ }^{2}$ \\ 1 - Department of Biology, University of Konstanz, D-78457 Konstanz, Germany \\ 2 - Institute of Pharmaceutical Sciences, Albert-Ludwigs-University Freiburg, Albertstr 25, D-79104 Freiburg, Germany
}

Correspondence to Jennifer N. Andexer: jennifer.andexer@pharmazie.uni-freiburg.de

\begin{abstract}
Chorismate-converting enzymes are involved in many biosynthetic pathways leading to natural products and can often be used as tools for the synthesis of chemical building blocks. Chorismatases such as FkbO from Streptomyces species catalyse the hydrolysis of chorismate yielding (dihydro)benzoic acid derivatives. In contrast to many other chorismate-converting enzymes, the structure and catalytic mechanism of a chorismatase had not been previously elucidated. Here we present the crystal structure of the chorismatase FkbO in complex with a competitive inhibitor at $1.08 \AA$ resolution. FkbO is a monomer in solution and exhibits pseudo-3-fold symmetry; the structure of the individual domains indicates a possible connection to the trimeric RidA/YjgF family and related enzymes. The co-crystallised inhibitor led to the identification of FkbO's active site in the cleft between the central and the C-terminal domains. A mechanism for FkbO is proposed based on both interactions between the inhibitor and the surrounding amino acids and an FkbO structure with chorismate modelled in the active site. We suggest that the methylene group of the chorismate enol ether takes up a proton from an active-site glutamic acid residue, thereby initiating chorismate hydrolysis. A similar chemistry has been described for isochorismatases, albeit implemented in an entirely different protein scaffold. This reaction model is supported by kinetic data from active-site variants of FkbO derived by sitedirected mutagenesis.
\end{abstract}

\section{Introduction}

Chorismate is a central metabolite in bacteria, plants and fungi and serves as key intermediate for biosynthesis of aromatic amino acids, quinones, salicylic acid and complex natural products such as polyketides, polyenes or terpenoids [1-3]. Moreover, many enzymes that catalyse reactions starting from chorismate (Fig. 1) are useful tools in the production of structurally diverse valuable, often chiral building blocks [4-6]. Knowledge of the three-dimensional structure of an enzyme usually greatly aids the understanding of the enzyme's catalytic mechanism. Although the crystal structures of various chorismateconverting enzymes have already been elucidated, the structure and mechanism of chorismatases is not yet known $[7,8]$. Some of the already extensively described enzymes share similarities in amino acid sequence, structure and mechanism such as enzymes from the isochorismate synthase family encompassing, for example, anthranilate synthase and salicylate synthase $[9,10]$. Similarities in the general mechanism have been proposed for enzymes such as chorismate mutase [11] and chorismate lyase [12] that catalyse different pericyclic reactions [13].

Chorismatases (Fig. 1) catalyse the hydrolysis of chorismate into pyruvate and (dihydro)benzoic acid derivatives. Depending on the benzoic acid derivative formed, three distinct types of chorismatases have been described: FkbO, Hyg5 and XanB2. Enzymes of the FkbO type catalyse the reaction leading to 3,4-dihydroxycyclohexa-1,5dienoic acid (3,4-CHD); all known enzymes of this type are involved in the biosynthetic pathways forming 


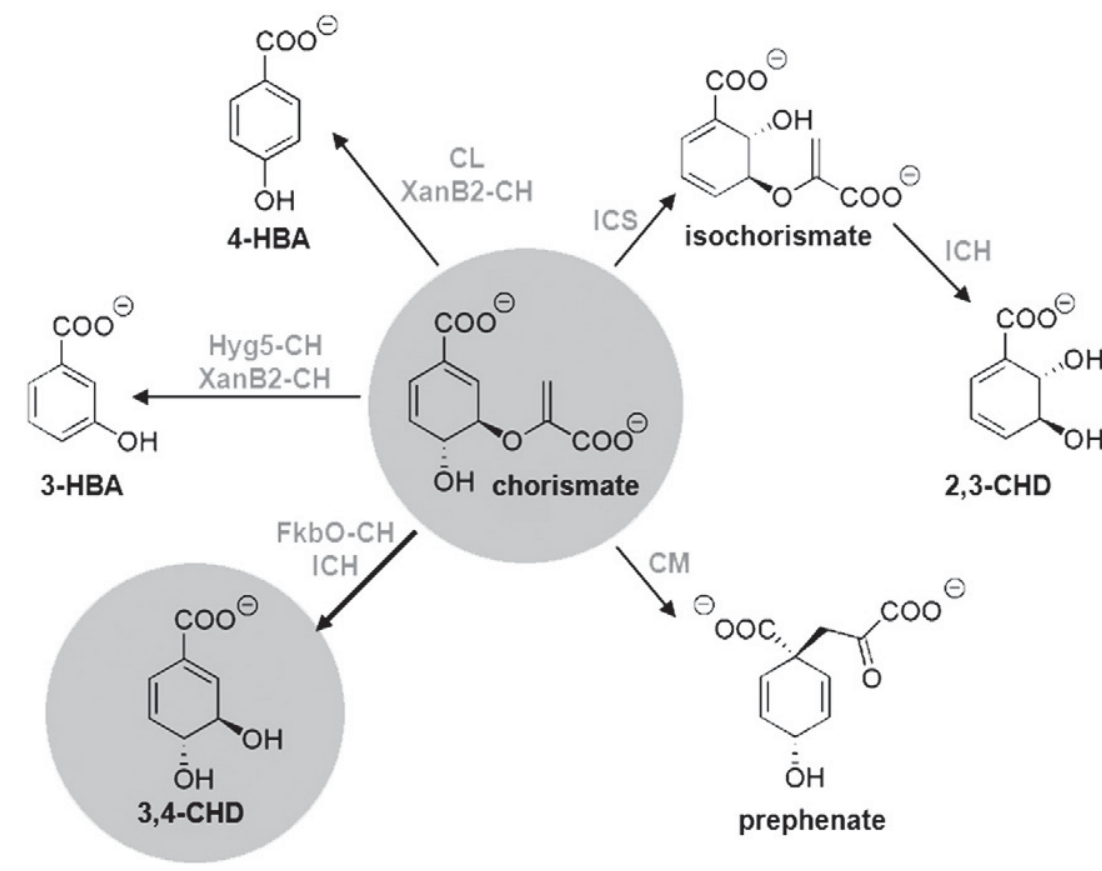

Fig. 1. Selected reactions catalysed by chorismatases and other chorismate-converting enzymes. Chorismatases (or chorismate hydrolases, $\mathrm{CH}$ ) of the FkbO-type catalyse chorismate hydrolysis leading to 3,4-dihydroxycyclohexa-1,5-dienoate (3,4-CHD); whereas Hyg5-type $\mathrm{CH}$ s result in the aromatic product 3-hydroxybenzoate (3-HBA). 3-HBA is also the main product of XanB2-type CHs, in addition to a chorismate lyase (CL) side activity yielding 4-hydroxybenzoate (4-HBA). Chorismate is isomerised by isochorismate synthases (ICS) to isochorismate; chorismate mutases (CM) catalyse the Claisen rearrangement to prephenate. Isochorismatases (or isochorismate hydrolases, ICH) that act on isochorismate leading to 2,3-dihydroxycyclohexa-4,6-dienoic acid (2,3-CHD) also catalyse a FkbO-type reaction.

polyketidic immunosuppressants such as ascomycin (FK520) [14], rapamycin [15] and tacrolimus (FK506) [16]. The second and the third type of chorismatases (Hyg5 type and XanB2 type) form the aromatic product 3-hydroxybenzoic acid (3-HBA). Hyg5 was cloned from a yet uncharacterised gene cluster from Streptomyces hygroscopicus [17], and Hyg5-like activity has been shown for the Bra8 enzyme from Nocardia brasiliensis [18] in in vivo experiments [7]. Very recently, the discovery of a Hyg5-type chorismatase leads to the identification of the gene cluster for cuevaene A in Streptomyces sp. LZ35 [19]. XanB2 from Xanthomonas sp. was described as the first member of a third type of chorismatases, characterised by the occurrence of Hyg5-like activity (3-HBA production) and chorismate lyase activity, leading to 4-hydroxybenzoic acid (4-HBA) production (Fig. 1). Sequence alignment studies and mutational analysis of conserved residues have been carried out with this enzyme, leading to a proposed model of the organisation of XanB2 in three domains, presenting distinct active sites for 3-HBA and 4-HBA production, respectively [8]. However, due to the lack of high-resolution structural data, no mechanism for the different types of chorismatases has yet been proposed.
An FkbO-type chorismatase side activity has been described for isochorismatases (E.C. 3.3.2.1), which catalyse the conversion of the chorismate regioisomer isochorismate into 2,3-dihydroxycyclohexa-4,6dienoic acid (Fig. 1) [20,21]. The three-dimensional structures of several isochorismatases are known [21-23], but the amino acid sequence identity shared with chorismatases is very low $(<10 \%)$; therefore, a structural relation between the two groups of enzymes can be neither assumed nor excluded.

Among experimentally identified and putative chorismatases, the amino acid sequences are very similar (35-84\% identity, see also Fig. S1); however, in comparison to other enzymes, the sequence similarity is very limited. Only the C-terminal domain of all chorismatases displays amino acid sequence similarity to the RidA (formerly YjgF/YER057c/UK114) protein family $(>22 \%$ identity). These proteins are abundant in bacteria, animals and fungi and were discussed to be involved in the regulation of translation processes and biosynthetic pathways and in the removal of potentially toxic metabolites [24-26]. Recently, a conserved enzymatic function for these proteins has been proposed, according to which they are responsible for the release of ammonia from reactive imine/enamine intermediates $[27,28]$. 
Structurally, RidA/YjgF proteins are related to $\mathrm{AroH}$ chorismate mutases catalysing the Claisen rearrangement of chorismate to prephenate $[11,13,29,25]$ : this connection to chorismate mutases initially led to the idea to test FkbO for chorismatase activity [7]. However, the stretch of sequence covered by the similarity to the RidA/YjgF family is not long enough to create a useful sequence-based homology model of chorismatases. Therefore, an experimental threedimensional structure of a chorismatase is of great value to draw conclusions about the catalytic mechanism and the distinction between the different types of chorismatases. Additionally, a protein structure would facilitate further comparisons with other chorismate-converting enzymes such as chorismate mutase, chorismate lyase or isochorismatase.

Here we report the crystal structure of the chorismatase FkbO from S. hygroscopicus subsp. ascomyceticus co-crystallised with the competitive inhibitor 3-(2-carboxyethyl)benzoate bound in the active site to a resolution of $1.08 \AA$. In combination with results of active-site mutagenesis studies, we propose a catalytic mechanism for FkbO.

\section{Results and Discussion}

\section{Oligomeric state and crystallisation of FkbO}

The sequence similarity search for FkbO using pBLAST (National Center for Biotechnology Information) revealed the RidA/YjgF protein family as the closest match. One of the main characteristics of these enzymes is their trimeric quaternary structure in solution that can also be found in case of the structurally similar AroH chorismate mutases. Initially, we thought that this might be the case for chorismatases as well; however, the analysis of the oligomeric state of FkbO with size-exclusion chromatography unequivocally showed that the active enzyme exists as a monomer in solution (Fig. S2). This finding was supported by the crystal structure of FkbO solved in space group $4(P 12,1)$ with one polypeptide in the asymmetric unit (Table 1). FkbO exclusively crystallised when 3-(2-carboxyethyl) benzoate was used as an additive, earlier crystallisation attempts without a ligand failed. The cocrystallised compound is a competitive inhibitor of FkbO (Table 2), mimicking the structure of the physiological substrate chorismate [30]. Attempts to solve the crystal structure based on molecular replacement with the RidA/YjgF protein family were unsuccessful. Therefore, experimental phases were determined by selenomethionine (SeMet) single wavelength anomalous dispersion. SeMet-FkbO crystals diffracted to $1.08 \AA$ allowing to model the structure with an $R$-factor of 0.126 and an $R$-free of 0.146 (Table 1). In the electron density map, the $13 \mathrm{~N}$-terminal amino acids of the native FkbO
Table 1. Crystallographic details

\begin{tabular}{|c|c|}
\hline \multicolumn{2}{|l|}{ Data collection } \\
\hline Protein sample & SeMet labelled \\
\hline Wavelength $(\AA)$ & 0.97794 \\
\hline Resolution range $(\AA)$ & $37.011 .081(1.1191 .081)$ \\
\hline \multirow{2}{*}{\multicolumn{2}{|c|}{ Unit cell length $(\AA)$}} \\
\hline & \\
\hline a & 49.139 \\
\hline$b$ & 52.25 \\
\hline$c$ & 52.532 \\
\hline \multicolumn{2}{|l|}{ Unit cell angle $\left(^{\circ}\right)$} \\
\hline$\alpha$ & 90 \\
\hline$\beta$ & 93.35 \\
\hline y & 90 \\
\hline Total reflections & $320,832(9926)$ \\
\hline Unique reflections & $103,504(5347)$ \\
\hline Multiplicity & $3.1(1.9)$ \\
\hline Completeness (\%) & $91.24(47.24)$ \\
\hline$R$-merge & $0.05953(0.2442)$ \\
\hline$R$-meas & 0.07132 \\
\hline Mean I/sigma (I) & $13.80(3.77)$ \\
\hline Wilson $B$-factor $\left(\AA^{2}\right)$ & 6.95 \\
\hline \multicolumn{2}{|l|}{ Refinement statistics } \\
\hline $\mathrm{CC}_{1 / 2}$ & $0.997(0.868)$ \\
\hline $\mathrm{CC}^{\star}$ & $0.999(0.964)$ \\
\hline$R$-work & $0.126(0.202)$ \\
\hline$R$-free & $0.146(0.205)$ \\
\hline \multicolumn{2}{|l|}{ Number of atoms } \\
\hline Protein & 2553 \\
\hline Ligand & 14 \\
\hline Water & 611 \\
\hline Protein residues & 331 \\
\hline r.m.s.d. bond lengths $(\AA)$ & 0.010 \\
\hline r.m.s.d. angles $\left({ }^{\circ}\right)$ & 1.45 \\
\hline Ramachandran favoured (\%) & 98.8 \\
\hline Ramachandran allowed (\%) & 1.2 \\
\hline Ramachandran outliers (\%) & 0 \\
\hline & 1.98 \\
\hline \multicolumn{2}{|l|}{ Average $B$-factor $\left(\AA^{2}\right)$} \\
\hline Protein & 9.20 \\
\hline Ligand & 5.30 \\
\hline Water & 22.30 \\
\hline
\end{tabular}

Statistics for the highest-resolution shell are given in parentheses.

sequence could not be modelled. N-terminal proteolytic truncation seems to be involved because of two further observations: (a) in a Western blot of an FkbO sample that was stored for 30 days at $4{ }^{\circ} \mathrm{C}$, the $\mathrm{N}$-terminal His-tag present in recombinant FkbO could not be detected with HisTag antibodies (Fig. S3); (b) crystals appeared only after extended periods, typically 4 weeks, in which proteolysis could occur. The $\mathrm{N}$-terminal proteolysed FkbO shows chorismatase activity comparable to full-length $\mathrm{FkbO}$ (Fig. S3); therefore, the N-terminal part does not seem to be involved in catalysis.

\section{The FkbO monomer is organised in three domains}

FkbO can be divided into three distinct domains: $\mathrm{N}$-terminal (residues 1-101), central (residues 102213) and C-terminal (residues 214-344; Figs. 2 and 3) domains. Each of the domains features a $\beta$-sheet with a high content of hydrophobic and 
Table 2. Activity of recombinant FkbO (wild type and variants) and inhibition by 3-(2-carboxyethyl)benzoate

\begin{tabular}{|c|c|c|c|}
\hline & \multirow{2}{*}{$\begin{array}{l}\text { Activity } \\
(\% \mathrm{wt})^{\mathrm{a}, \mathrm{b}}\end{array}$} & \multicolumn{2}{|c|}{ Kinetic parameters $^{\mathrm{b}}$} \\
\hline & & $\begin{array}{c}K_{\mathrm{M}}, S_{0.5^{c}}, K_{\mathrm{i}}^{\mathrm{d}} \\
(\mathrm{mM})\end{array}$ & $\begin{array}{c}V_{\max } \\
(\mu \mathrm{mol} / \mathrm{min} / \mathrm{mg})\end{array}$ \\
\hline $\begin{array}{l}\text { FkbO wild type } \\
\text { FkbO + inhibitor }\end{array}$ & $\begin{array}{l}100 \\
10.9^{\mathrm{e}}\end{array}$ & $\begin{array}{l}0.15 \pm 0.02 \\
1.02 \pm 0.22^{d}\end{array}$ & $0.56 \pm 0.02$ \\
\hline FkbO-Tyr215Phe & 12 & $0.66 \pm 0.24$ & $0.06 \pm 0.01$ \\
\hline FkbO-Phe226Ala & 2.5 & n.d. ${ }^{f}$ & n.d. ${ }^{f}$ \\
\hline FkbO-Arg228Alac & 224.5 & $0.21 \pm 0.01$ & $0.87 \pm 0.02$ \\
\hline FkbO-Glu338Ala & 0 & Inactive & Inactive \\
\hline FkbO-Glu338Gln & 0 & Inactive & Inactive \\
\hline
\end{tabular}

a Residual activity (compared to wild type) using standard assay conditions [100 mM Tris $\mathrm{HCl}(\mathrm{pH} 7.0), 1 \mathrm{mM}$ chorismate, $100 \mu \mathrm{g} / \mathrm{mL}$ enzyme, $5 \mathrm{mg} / \mathrm{mL} \mathrm{NADH}, 25 \mathrm{U} / \mathrm{mL}$ lactate dehydrogenase; the decrease of NADH concentration was followed for $5 \mathrm{~min}$ at $340 \mathrm{~nm}$ and $25^{\circ} \mathrm{C}$ ].

b Mean values derived from three independent experiments.

c Kinetic parameters have been calculated using the Hill equation resulting in a Hill coefficient of $n \quad 2.11 \pm 0.23$.

${ }_{\mathrm{d}} K_{\mathrm{i}}$ value determined for 3-(2-carboxyethyl)benzoate [30].

e Data from $\mathrm{IC}_{50}$ determination using a competitive assay with $0.2 \mathrm{mM}$ chorismate and $20 \mathrm{mM}$ 3-(2-carboxyethyl)benzoate (concentration used in crystallisation experiments).

${ }^{f}$ n.d., not determined: due to the low activity and the largely reduced substrate affinity, a useful determination of kinetic parameters was not always possible.

aromatic amino acid residues forming the inner core of the protein. The three individual domains and the complete structure were analysed for similar structures using the DALI server [31]. The results exclusively show similarities to the RidA/YjgF protein family and $\mathrm{AroH}$ chorismate mutases that are characterised by a mostly anti-parallel $\beta$-sheet arranged in an $\beta 1-\beta 2-\beta 3-\beta 6-\beta 4-\beta 5$ order, with two $\alpha$-helices located between $\beta 3$ and $\beta 4$ and between $\beta 4$ and $\beta 5$, respectively (Fig. 3a and Fig. S4) [25-27]. These similarities are most striking for the C-terminal domain, where the relation is also obvious from sequence alignments; in the structure, the complete six-stranded $\beta$-sheet is preserved, including the inserted $\alpha$-helices, although one of them is represented by two shorter helices that are connected by a helical loop region. The structural relation of RidA/ YjgF with the FkbO central domain was not predicted by sequence alignments; however, it shows a complete RidA/YjgF fold that only lacks the first $\beta$-strand. The $\mathrm{N}$-terminal domain has less obvious structural similarity to the RidA/YjgF motif, as no a-helices are present. Nevertheless, the underlying topology of the core $\beta$-sheet is the same, with four $\beta$-strands present, which are connected by loop regions corresponding to the position of the helices in RidA/YjgF proteins (Fig. 3a and Fig. S4). Overall, the $\mathrm{FkbO}$ monomer resembles the trimers of the RidA/YjgF family and $\mathrm{AroH}$ chorismate mutase proteins (Fig. 3b). The similarity between the central and the C-terminal domains of FkbO suggests their origin from a gene duplication event. The sequence identity between the two domains is low (12\%); thus, the shared origin mostly manifests in the structure [32]. The lower similarity of the $\mathrm{N}$-terminal domain to the central and C-terminal domains makes their relation through another gene duplication event less obvious. In context with their XanB2 work, Zhou et al. suggest based on sequence alignments that the variability of the N-terminal part of chorismatases might be the main discrimination between the different chorismatase types [8]. If this is the case, the differing appearance of the $\mathrm{N}$-terminal domain might be connected to the development of new functionalities. As it is far away from the active site and apparently not involved in the catalysis (discussion of the mechanism below), it might have been less restrained during evolution and could therefore have evolved to assume new functions as described for XanB2 (chorismate lyase activity).

Interestingly, a similar concatenation of RidA/YjgF monomers leading to a pseudo-3-fold symmetry (coined the "Toblerone" fold) has been recently described for the cyanuric acid hydrolase AtzD, which belongs to a family of ring-opening amidases [33]. Both enzymes, AtzD and FkbO, are unrelated, concerning the reaction catalysed and their amino acid sequence ( $7 \%$ overall sequence identity), but show modest structural similarity (r.m.s.d., 3.8 ). In case of AtzD, the single domains ("repeating units") show higher sequence identity $(9.3-17.8 \%)$ to each other than the domains in FkbO (N-terminal domain-central domain, $6.5 \%$; $\mathrm{N}$-terminal domainC-terminal domain, $5.9 \%$; central domain-C-terminal domain, $12.0 \%$ ), whereas the r.m.s.d. values for the single domains are in the same range: $2.2-3.0 \AA$ for AtzD and 2.4-2.8 $\AA$ for FkbO. In contrast to $\mathrm{FkbO}$, AtzD forms tetramers in solution.

The most obvious difference between FkbO and AtzD is the location of the active site, which is in case of AtzD located in the centre of the enzyme, with all three domains contributing to its structure. As described in the following, FkbO's single active site shows parallels with the active sites present in the trimeric RidA/YjgF proteins. In their paper, Peat et al. also favour the idea that AtzD and related enzymes are derived from RidA/YjgF family enzymes via gene duplication as opposed to RidA/YjgF developing from a truncated AtzD [33]. The existence of two different usages of a concatenated RidA/YjgF fold further supports this idea and suggests that the RidA/YjgF monomer is a most versatile domain architecture.

\section{FkbO's active site is located between the central and the C-terminal domains}

Only one molecule of the inhibitor 3-(2-carboxyethyl) benzoate is found in the FkbO structure: the compound is located in the cleft between the central and the C-terminal domains, suggesting only one active site 
(a)
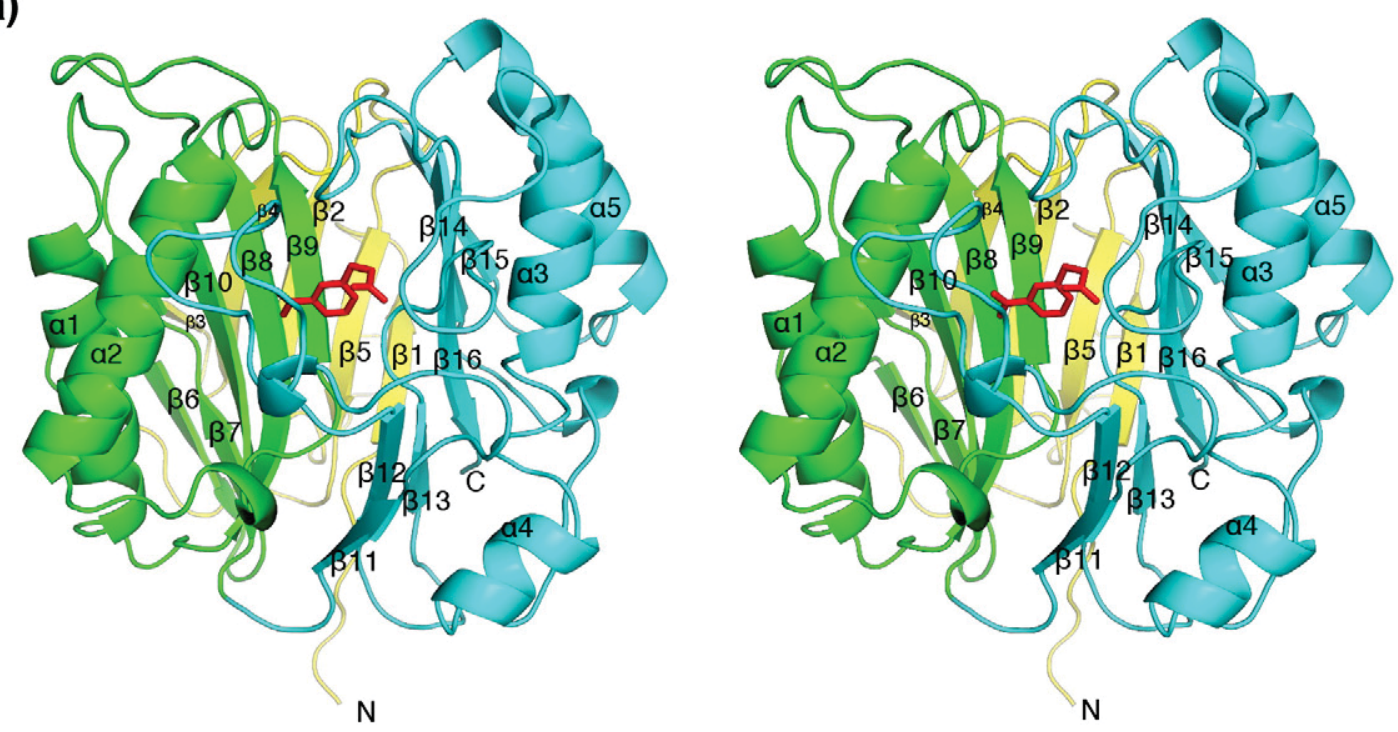

(b)

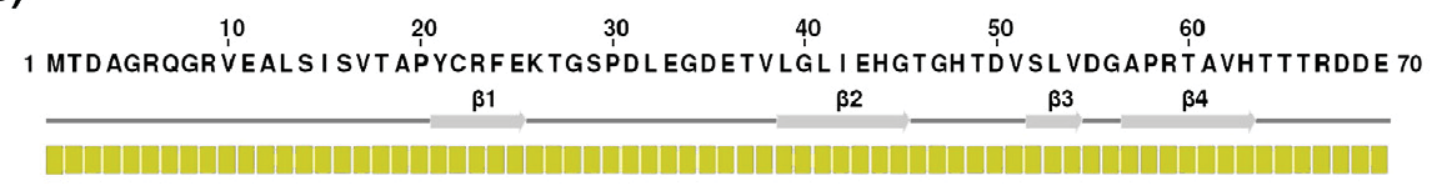

$800100 \quad 90 \quad 110 \quad 120 \quad 130$

71 AFTEVWHAQR PVESGMDNG I AWARTDAYLFGVVRTGESGR YADATAAL YTNVFQLTRSLGYPLLARTWNY 140 $\beta 5$ $\beta 6$ $\beta 7$

$\alpha 1$ $\beta 8$

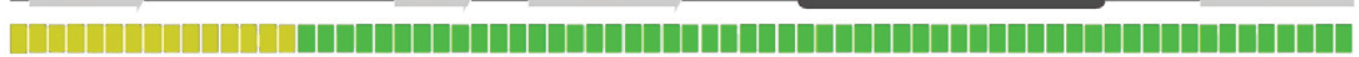
150 160 170 180 190 200 141 VSG INTTNADGLEVYRDFCVGAQALDEGG I DPATPAATG I GAHGGG I TCVFLAARGGVR IN I ENPAVL 210 a2 $\beta 9$ $\beta 10$ $\beta 11 \ldots \ldots$ (1) $2202230 \quad 240 \quad 250 \quad 260 \quad 270$ 211 TAHHYPTTYGPRPPVAATWLGPPEGGRLFI SATAG I LGHRTVHHGDVTGQCEVALDNMAR VIGAENLR 280 a3 a4

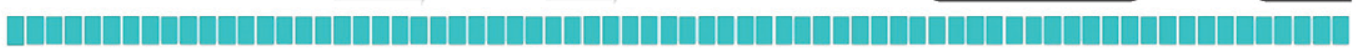

281 RHGVQRGHVL ADVDHLKVYVRRREDLDTVRRVCAARLSST AAVALLHTD I ARED LLV I I I EGMVA

Fig. 2. Structure and sequence of FkbO. (a) Stereo view of FkbO (cartoon representation) in complex with the inhibitor 3-(2-carboxyethyl)benzoate (red sticks). The enzyme is clearly divided in three domains (N-terminal domain, yellow; central domain, green; C-terminal domain, blue), and the inhibitor is bound between the central and the C-terminal domains. (b) Sequence of FkbO illustrating the domain organisation (colours as above), secondary structure elements and residues suggested to be active in catalysis and substrate/inhibitor binding (highlighted in red). The loops covering the active site are delimited with blue dots. 
(a)

\begin{tabular}{|c|c|c|c|c|}
\hline \multirow[t]{2}{*}{ () } & \multirow{2}{*}{$\begin{array}{l}\text { RutC monomer } \\
\text { RidA/YjgF fold }\end{array}$} & \multicolumn{3}{|c|}{ FkbO domains } \\
\hline & & N-terminal & central & C-terminal \\
\hline $\begin{array}{l}\beta \text {-sheet } \\
\text { topology }\end{array}$ & $\begin{array}{c}\beta 1-\beta 2-\beta 3-\beta 6- \\
\beta 4-\beta 5\end{array}$ & $\begin{array}{c}\beta 1-\beta 5-\beta 2-\beta 4 \\
(-\beta 3)\end{array}$ & $\begin{array}{c}\beta 6-\beta 7-\beta 10-\beta 8- \\
\beta 9\end{array}$ & $\begin{array}{c}\beta 11-\beta 12-\beta 13-\beta 16- \\
\beta 14-\beta 15\end{array}$ \\
\hline a-helices & $\begin{array}{l}(\beta 3-) \alpha 1(-\beta 4) \\
(\beta 4-) \alpha 2(-\beta 5)\end{array}$ & no a-helices & $\begin{array}{l}(\beta 7-) \alpha 1(-\beta 8) \\
(\beta 8-) \alpha 2(-\beta 9)\end{array}$ & $\begin{array}{c}(\beta 13-) \alpha 3-\alpha 4(-\beta 14) \\
(\beta 14-) \alpha 5(-\beta 15)\end{array}$ \\
\hline 3D structure & & & & \\
\hline \multirow{2}{*}{$\begin{array}{l}\text { Results of DAL } \\
\text { analysis (3V4D) }\end{array}$} & r.m.s.d. (A) & 3.1 & 1.9 & 1.6 \\
\hline & Z-score & 2.5 & 9.7 & 16.6 \\
\hline
\end{tabular}

(b)

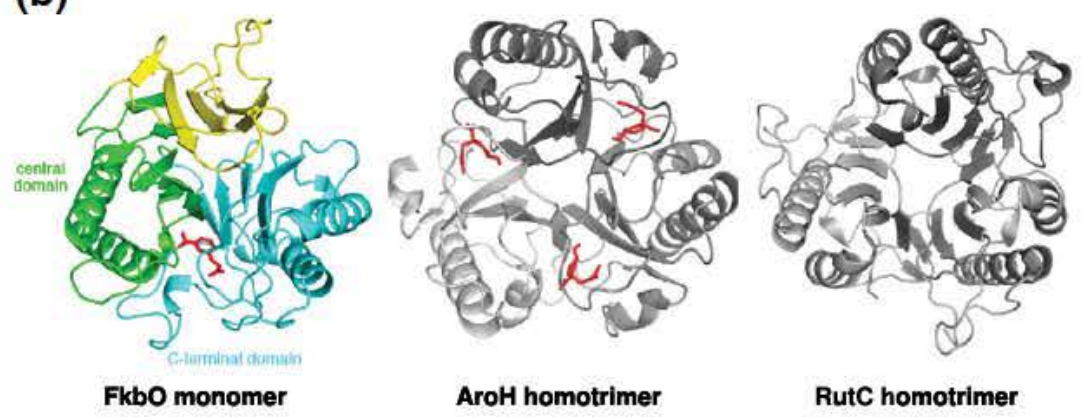

Fig. 3. FkbO in comparison with the RidA/YjgF/AroH fold. (a) Topology of FkbO domains and the RutC monomer (RidA/ YjgF family). RutC was also used in structural comparison with the DALI server. Corresponding secondary structure elements are colour-coded as follows: $\beta 1-\beta 2$, grey; $\beta 3$, blue; $\alpha 1$, cyan; $\beta 4$, green; $\alpha 2$, yellow; $\beta 5$, orange; $\beta 6$, red. In the $\mathrm{N}$-terminal domain, the helices are replaced by loops and an additional perpendicularly arranged $\beta$-strand. In the C-terminal domain, the first $\alpha$-helix corresponds to two shorter helices that are connected by a loop region. $\beta 1-\beta 2$ and $\beta 1$ are missing in the $\mathrm{N}$-teminal and in the central domains, respectively. See also Fig. S4 for individual overlays of the RutC monomer with the single FkbO domains. (b) Top view of FkbO. Organisation of the FkbO monomer in three domains: the active site is located between the central and the C-terminal domains (left). Top view of the RidA/YjgF/AroH fold: the trimer is made up from three identical monomers; the active sites are located at each interface \{middle, AroH chorismate mutase (PDB entry 1COM) [11]; right, RidAYjgF protein RutC (PDB entry 3V4D) [24]\}.

(Figs. 2a and 3b). Since 3-(2-carboxyethyl)benzoate is a competitive inhibitor [30], it is likely that the compound binds to the active site of the enzyme in a similar fashion as the native substrate chorismate. As obvious from the surface representation (Fig. S5), the inhibitor is almost completely enclosed inside the FkbO molecule; the entrance to the active site is covered by three loops from the C-terminal domain (Fig. 4a). Loop 1 [composed of 23 residues (206-228)] contains five proline residues most of which are conserved in chorismatases (Fig. S1). The conformational rigidity of proline residues may reduce the conformational flexibility of the loop in absence of substrate. As a consequence, the entropy cost associated with freezing loop 1 into one conformation upon substrate binding would be reduced and the binding affinity would be enhanced [34].

3-(2-Carboxyethyl)benzoate interacts non-covalently with the loops and the secondary structure elements forming the cleft. The aromatic ring of the inhibitor is $\pi$ - $\pi$-stacked with Phe226 in loop 1 on one side and stabilised by water- $\pi$ interaction on the other side. The carboxylic group at the aromatic ring of the inhibitor forms two hydrogen bonds with the terminal amino groups of Arg162. Glu338 interacts with the $\mathrm{C} 2$ carbon atom of the propionate group, and two water molecules build a hydrogen bond network with the side-chain carboxyl group. Tyr215 and Arg228 are also hydrogen bonding with the side-chain carboxyl group (Fig. 4b). Structure-based 

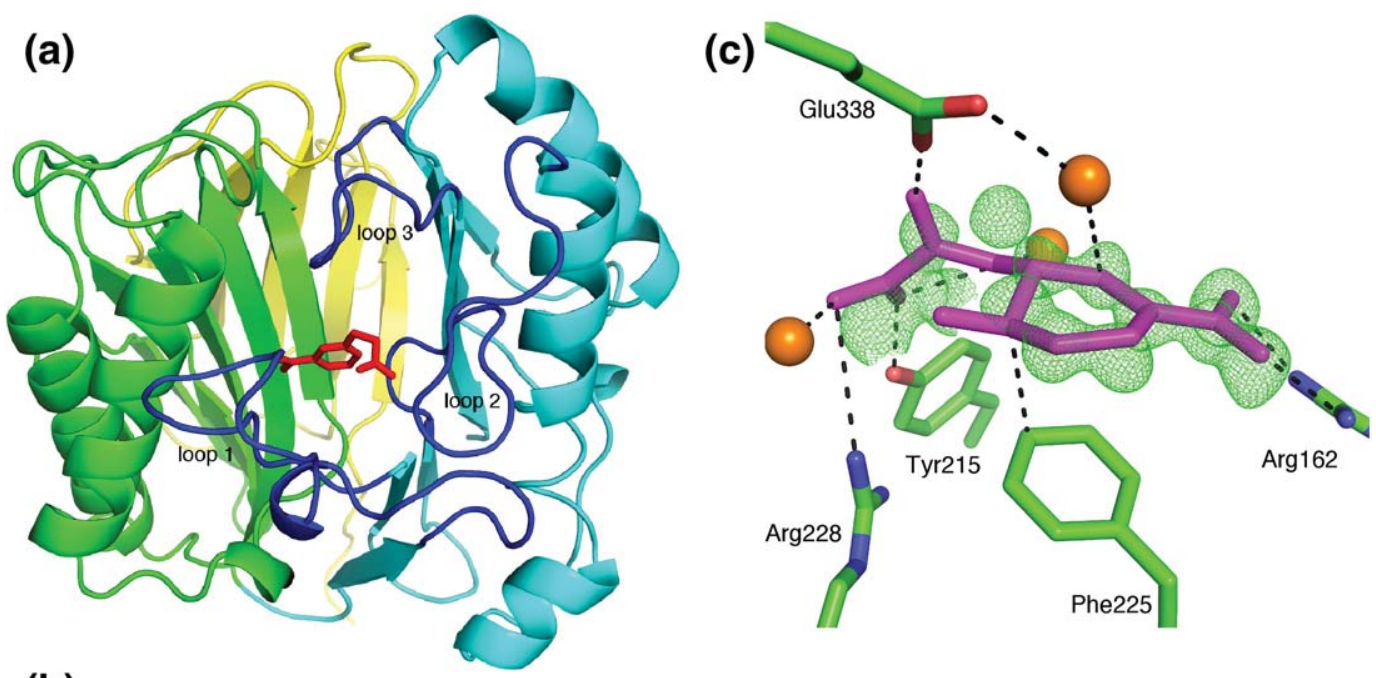

(b)

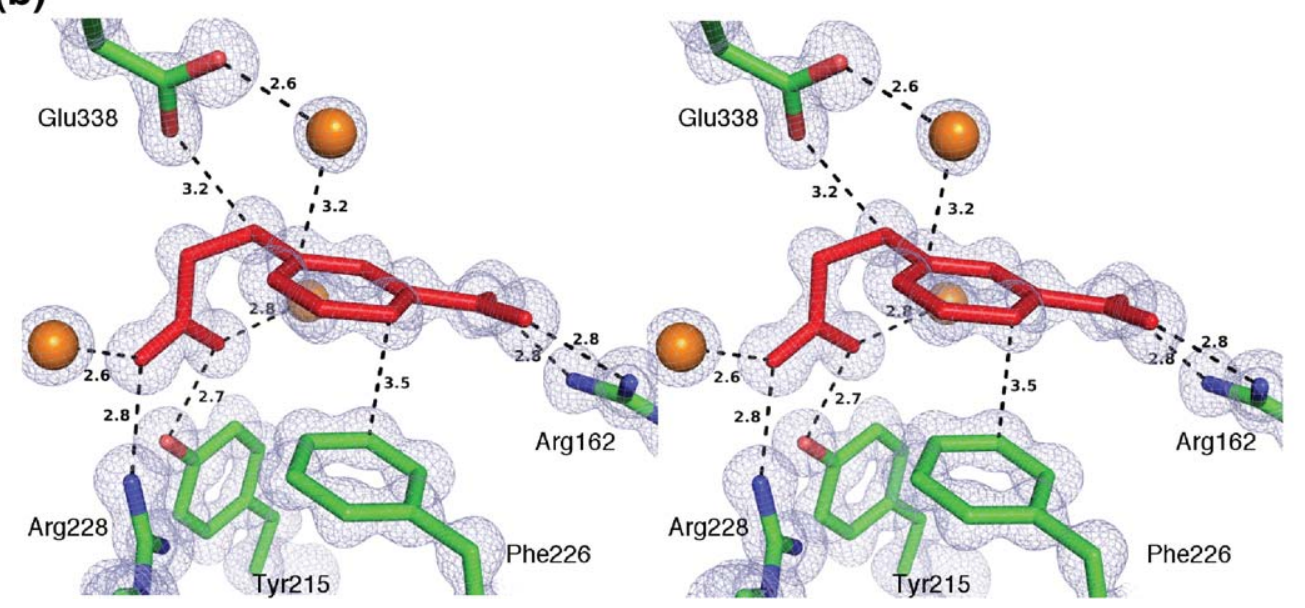

Fig. 4. Active site of FkbO. (a) Putative entrance to the active site. Three loops (highlighted in blue) cover the inhibitor 3-(2-carboxyethyl)benzoate. (b) Stereo view of the inhibitor 3-(2-carboxyethyl)benzoate in the active site and 2mFo-DFc map contoured to a sigma level of 1.5. Hydrogen bonds interacting with the inhibitor are indicated with broken lines. Orange spheres are water molecules. (c) The natural substrate chorismate (in the pseudo-diequatorial conformation) modelled in the active site, based on the mFo-DFc map for the inhibitor 3-(2-carboxyethyl)benzoate created by simulated annealing (map contoured at a sigma level of 3.0), and possible interactions of the substrate with active-site residues are denoted with broken lines.

alignments of FkbO-type chorismatases show that all these residues are conserved with the exception of Arg228 that is exchanged by glycine in the chorismatase RapK (Fig. S1).

\section{A putative mechanism for FkbO-catalysed chorismate hydrolysis}

The electron density of the inhibitor served as a template to model the native substrate chorismate. In the model, chorismate is stabilised by the same amino acids described for the inhibitor (Phe226, Arg162, Tyr215, Arg228), with the exception that Glu338 interacts with the $\mathrm{CH}_{2}$ group of the enol ether (absent in the inhibitor) (Fig. 4c). Based on this observation, the following mechanism for FkbO could be proposed: after entering the active site, the methylene group of the enol ether takes up a proton from Glu338 forming a carbocation/oxocarbonium ion intermediate. This species is subsequently hydroxylated resulting in a hemiketal intermediate, which spontaneously decomposes to the products 3,4-CHD and pyruvate (Fig. 5). Additional amino acid residues might be involved in the activation of the water molecule hydroxylating the cationic intermediate and in protonation of the hemiketal intermediate. A similar mechanism has been postulated for the isochorismatase PhzD from Pseudomonas aeruginosa [21] and an isochorismatase-like hydrolase from Oleispira antarctica [23]. However, there is no structural similarity between the FkbO structure and the dimeric $\alpha-\beta-\alpha$ sandwich structure described for 


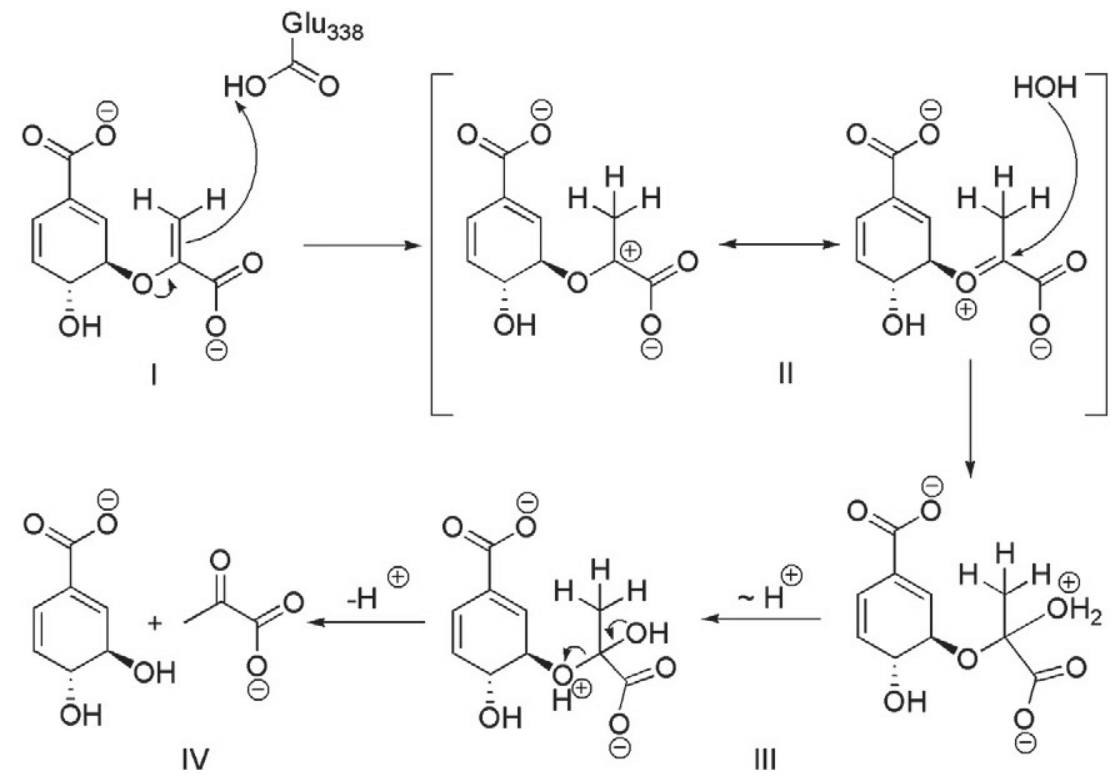

Fig. 5. Suggested mechanism for FkbO. After binding of the substrate, the enol ether methylene group takes up a proton from the catalytic residue Glu338 (I). The resulting carbocation/oxocarbonium ion (II) intermediate is subsequently hydroxylated resulting in a hemiketal intermediate (III) that spontaneously decomposes into the products 3,4-CHD and pyruvate (IV). For details, see the main text.

isochorismatases. For other chorismate-converting enzymes such as chorismate mutases or isochorismatases, it has been observed that chorismate (or isochorismate) is bound in the active site in the trans pseudo-diaxial conformation; for chorismate mutases, this conformation is the basis for catalysis $[21,35]$. The pseudo-diaxial conformer is the minor species in solution, as the pseudo-diequatorial conformation is shown to be the more stable one [36]. With the current model (the pseudo-diequatorial conformation is shown in Fig. 4c), we cannot rule out one of the two conformations; however, in light of the mechanistic similarity between $\mathrm{FkbO}$ and isochorismatases, the trans pseudo-diaxial conformation might be present in chorismatases as well.

To further support the postulated chorismatase mechanism, we tested several active-site mutants in an enzyme-coupled, continuous spectrophotometric assay (Table 2 and Figs. S6 and S7). The results obtained confirm that Glu338 plays a major role in catalysis, as in both enzyme variants tested (FkbOGlu338Ala and the more conserved replacement Glu338GIn), chorismatase activity is completely abolished. This finding was confirmed by HPLC: even after incubation of the variants with chorismate for $20 \mathrm{~h}$, no 3,4-CHD could be detected (Fig. S7). Studies of Zhou et al. further support these results, as the mutation of the corresponding amino acid in XanB2 (Glu329) severely affects XanB2-chorismatase activity (43\% residual activity for 3-HBA formation) [8]. In this case, the glutamic acid was exchanged with aspartic acid; therefore, catalytic function is retained at reduced activity and only the relative position of the carboxyl group to the substrate is changed, explaining the significant residual activity for the XanB2 variant. Exchange of the amino acid stabilising the substrate through $\pi-\pi$ interaction (Phe226Ala) and the tyrosine hydrogen bonding with the side-chain carboxyl group (Tyr215Phe) also led to FkbO variants with decreased activities $(2.5 \%$ and $12 \%$ remaining wildtype activity, respectively). Surprisingly, the Arg228Ala variant showed an approximately doubled activity compared to the wild type, along with a clearly S-shaped substrate concentration/activity plot suggesting cooperativity. The non-linear fit of the curve with the Hill equation results in a Hill coefficient of 2.1. More detailed experiments will be carried out to explore potential reasons for this result. Interestingly, Arg228 is the only active-site amino acid not present in all FkbO-type chorismatases; in the chorismatase from the rapamycin cluster (RapK), a glycine is present at this position (Fig. S1). The decreased activity of Tyr215Phe suggests that Tyr215 is the main amino acid residue interacting with the side-chain carboxyl group.

\section{Comparison of the active sites from FkbO and RidA/YjgF proteins}

The finding that $\mathrm{FkbO}$ has only one active site is in contrast to the situation in RidA/YjgF family proteins and AroH chorismate mutases. Due to their symmetrical arrangement of three monomers, they have three ligand binding sites per trimer $[11,13,25,26]$. 
When FkbO and RidA/YjgF protein structures containing active-site ligands are compared, it is obvious that the ligands and interacting active-site residues superimpose (Fig. S5). In the RidA/YjgF family, the glutamate corresponding to the catalytic Glu338 in FkbO is largely conserved and is suggested to coordinate the water molecule that reacts with the imine/enamine substrate [28]. Another feature conserved in the active sites of both, RidA/ YjgF proteins and chorismatases, is the aromatic residue (Phe226 in FkbO) forming $\pi-\pi$ interaction with the substrate in $\mathrm{FkbO}$. This residue is a tyrosine in most RidA/YjgF proteins and proposed to stabilise the imine nitrogen $[27,28]$. Other residues suggested to participate in catalysis or substrate binding in RidA/YjgF proteins and FkbO are not conserved (Fig. S5). Nevertheless, the similarities in the active site strongly support the idea that RidA/YjgF proteins and chorismatases share a common ancestor, as it has been suggested for RidA/YjgF proteins and chorismate mutases or the AtzD family, respectively $[25,33]$.

When compared to AroH chorismate mutases, FkbO shows no striking similarities except the overall structure and the general location of the active site at the monomer-monomer interfaces, as well as the common fact that both catalyse reactions starting from chorismate. AroH chorismate mutases might therefore be distinct relatives of chorismatases, sharing the same substrate but evolved divergently to remain homotrimeric enzymes with three active sites rather than to become a monomeric enzyme with only one active site. Further experiments may clarify whether the utilisation of slightly different domains as opposed to three identical monomers is the basis for chorismatase activity or whether the inactive domains serve as regulatory elements. For XanB2, it has already been shown that different domains are responsible for different activities (chorismatase versus chorismate lyase activity) [8].

In summary, we believe that the structure presented will be a step towards a molecular understanding of the different chorismatase types (FkbO, Hyg5, XanB2) and their relation to other chorismate-converting enzymes. Due to the involvement of chorismate-converting enzymes in the biosynthetic pathways of diverse natural products and, therefore, their importance as targets for new pharmaceuticals, a detailed knowledge of the catalytic mechanism aids the development and improvement of compounds by the design of specific inhibitors or enzyme variants leading to natural product derivatives with enhanced bioactivity.

\section{Experimental Procedures}

Details of synthetic procedures, cloning, expression and purification of FkbO variants and enzyme assays are given in Supplemental Information.

\section{Chemicals}

All chemicals used were of analytical grade and, if not stated otherwise, used as received from the supplier. 3-(2-Carboxyethyl)benzoate was synthesised via Knoevenagel-Doebner condensation followed by reduction of the double bond and subsequent transformation in the corresponding sodium salt as described before [30].

\section{Heterologous expression and purification of FkbO and FkbO variants}

Heterologous expression and purification of recombinant FkbO (from $S$. hygroscopicus subsp. ascomyceticus) using the strain BL21-RPpL1SL2(DE3)-pET28a-FkbO (carrying an N-terminal His-tag) was carried out as described previously [30]. For further purification, the enzyme was subjected to size-exclusion chromatography (Superdex 75 10/300 GL; GE Healthcare) in $20 \mathrm{mM}$ sodium phosphate buffer ( $\mathrm{pH} 7.0)$, containing $100 \mathrm{mM} \mathrm{NaCl}$ and $1 \mathrm{mM}$ tris(2-carboxyethyl)phosphine.

\section{SeMet-FkbO}

We used $100 \mu \mathrm{L}$ of an overnight culture of BL21RP-pL1SL2(DE3)-pET28a-FkbO to inoculate a second overnight culture in a SeMet-containing minimal medium. Expression was carried out as described by Dias et al. [37] with an incubation time of $24 \mathrm{~h}$ at $18{ }^{\circ} \mathrm{C}$ and $200 \mathrm{rpm}$. The purification of SeMet-FkbO followed the same protocol as described for FkbO.

\section{FkbO variants}

Active-site variants were constructed with the QuikChange method and expressed using the same strain as described for wild-type FkbO; to improve yields, we carried out the heterologous expression at $24{ }^{\circ} \mathrm{C}$ for $20 \mathrm{~h}$. The purification procedure was the same as described for wild-type FkbO.

\section{Activity assays}

Chorismatase activity was determined as described before using a continuous spectrophotometric assay by following the decrease in NADH concentration with lactate dehydrogenase as a coupling enzyme; for the analysis of extended incubation times, an HPLC assay (reversed phase, C18 material) was employed [30]. Kinetic parameters were calculated with the Origin software using the Michaelis-Menten or the Hill equation.

\section{Crystallisation of FkbO and SeMet-FkbO}

Prior to crystallisation, the protein was concentrated to $4 \mathrm{mg} / \mathrm{mL}$ (spin filters, cutoff of $30 \mathrm{kDa}$ ) and 
afterwards supplemented with $5 \%$ glycerol and 20 mM 3-(2-carboxyethyl)benzoate. Both native and SeMet-labelled FkbO crystals were obtained via hanging-drop vapour diffusion at $18^{\circ} \mathrm{C}$ by equilibration against a reservoir solution of $0.1 \mathrm{M}$ 4-morpholineethanesulfonic acid buffer ( $\mathrm{pH} \mathrm{6.5)}$ and $20-25 \%$ polyethylene glycol 4000 (1:1 ratio of protein to reservoir solution). SeMet-FkbO crystals were obtained from SeMet-FkbO $(4 \mathrm{mg} / \mathrm{mL})$ by streak seeding using native FkbO crystals. Welldiffracting crystals were obtained after a period of 4 weeks.

\section{Data collection and phase determination}

Diffraction data were collected at the PXI(XO6SA) at the Swiss Lightsource, Paul Scherrer Institute in Villigen, Switzerland. Data reduction was carried out with the X-ray Detector Software (XDS Program Package) [38,39]. The structure was solved with phases obtained from SeMet-FkbO crystals via single wavelength anomalous dispersion using HKL2MAP [40].

\section{Model building and refinement}

A first model was built manually in Coot [41], and hydrogens were added in their riding positions. The preliminary model was iteratively completed within several rounds of rebuilding and runs of phenix.refine [42] ultimately refining all heavy atoms anisotropically. Ligand coordinates and restraints were generated using the Grade Web Server ${ }^{\ddagger}$. The structure was evaluated using the MolProbity server ${ }^{\S}$. Figures were prepared using PyMOL (PyMOL Molecular Graphics System, Version 1.2r3pre, Schrödinger, LLC).

\section{Protein coordinates}

Protein coordinates have been submitted to the PDB database with the accession code 4BPS.

\section{Acknowledgments}

P.J. would like to thank the Konstanz Research School Chemical Biology for financial support. J.N.A. and F.H. thank Prof. Dr. Michael Müller and Eduard Frick (University of Freiburg, Germany) for helpful discussions and Dr. Michael Richter (Empa, Switzerland) for critically reading the manuscript. Further, we would like to thank the Swiss Lightsource staff (Villingen, Switzerland) for their support during data collection.

\section{Appendix A. Supplementary data}

Supplementary data to this article can be found online at http://dx.doi.org/10.1016/j.jmb.2013.09.006.

Received 1 August 2013; Received in revised form 6 September 2013; Accepted 9 September 2013 Available online 13 September 2013

Keywords: chorismate; site-directed mutagenesis; high-resolution structure; RidA/YjgF; toblerone fold

\author{
${ }^{\dagger}$ P.J. and F.H. contributed equally to this work. \\ *http://grade.globalphasing.org \\ $\S$ http://molprobity.biochem.duke.edu
}

Abbreviations used:

3,4-CHD, 3,4-dihydroxycyclohexa-1,5-dienoic acid; 3-HBA, 3-hydroxybenzoic acid; 4-HBA, 4-hydroxybenzoic acid; SeMet, selenomethionine.

\section{References}

[1] Tzin V, Galili G. New insights into the shikimate and aromatic amino acids biosynthesis pathways in plants. Mol Plant 2010;3:956 72 .

[2] Dosselaere F, Vanderleyden J. A metabolic node in action: chorismate-utilizing enzymes in microorganisms. Crit Rev Microbiol 2001;27:75 131.

[3] Floss HG. Natural products derived from unusual variants of the shikimate pathway. Nat Prod Rep 1997;14:433 52.

[4] Bongaerts J, Esser S, Lorbach V, Al-Momani L, Müller MA, Franke D, et al. Diversity-oriented production of metabolites derived from chorismate and their use in organic synthesis. Angew Chem Int Ed 2011;50:7781 6.

[5] Müller R, Breuer M, Wagener A, Schmidt K, Leistner E. Bacterial production of trans-dihydroxycyclohexadiene carboxylates by metabolic pathway engineering. Microbiology 1996;142:1005 12.

[6] Sprenger GA. From scratch to value: engineering Escherichia coli wild type cells to the production of L-phenylalanine and other fine chemicals derived from chorismate. Appl Microbiol Biotechnol 2007;75:739 49.

[7] Andexer JN, Kendrew SG, Nur-e-Alam M, Lazos O, Foster TA, Zimmermann A-S, et al. Biosynthesis of the immunosuppressants FK506, FK520, and rapamycin involves a previously undescribed family of enzymes acting on chorismate. Proc Natl Acad Sci USA 2011;108:4776 81.

[8] Zhou L, Wang J-Y, Wang J, Poplawsky A, Lin S, Zhu B, et al. The diffusible factor synthase XanB2 is a bifunctional chorismatase that links the shikimate pathway to ubiquinone and xanthomonadins biosynthetic pathways. Mol Microbiol 2013;87:80 93.

[9] He Z, Stigers Lavoie KD, Bartlett PA, Toney MD. Conservation of mechanism in three chorismate-utilizing enzymes. J Am Chem Soc 2004;126:2378 85. 
[10] Kerbarh O, Ciulli A, Howard NI, Abell C. Salicylate biosynthesis: overexpression, purification, and characterization of Irp9, a bifunctional salicylate synthase from Yersinia enterocolitica. J Bacteriol 2005;187:5061 6.

[11] Chook YM, Gray JV, Ke H, Lipscomb WN. The monofunctional chorismate mutase from Bacillus subtilis: structure determination of chorismate mutase and its complexes with a transition state analog and prephenate, and implications for the mechanism of the enzymatic reaction. J Mol Biol 1994;240:476 500.

[12] Gallagher DT, Mayhew M, Holden MJ, Howard A, Kim K-J, Vilker VL. The crystal structure of chorismate lyase shows a new fold and a tightly retained product. Proteins 2001;44:304 11 .

[13] Lamb AL. Pericyclic reactions catalyzed by chorismateutilizing enzymes. Biochemistry 2011;50:7476 83.

[14] Wu K, Chung L, Revill WP, Katz L, Reeves CD. The FK520 gene cluster of Streptomyces hygroscopicus var. ascomyceticus (ATCC 14891) contains genes for biosynthesis of unusual polyketide extender units. Gene 2000;251:8190.

[15] Graziani El. Recent advances in the chemistry, biosynthesis and pharmacology of rapamycin analogs. Nat Prod Rep 2009;26:602 9.

[16] Motamedi H, Shafiee A. The biosynthetic gene cluster for the macrolactone ring of the immunosuppressant FK506. Eur $\mathrm{J}$ Biochem 1998;256:528 34

[17] Ruan X, Stassi D, Lax SA, Katz L. A second type-I PKS gene cluster isolated from Streptomyces hygroscopicus ATCC 29253, a rapamycin-producing strain. Gene 1997;203:1 9.

[18] Hayashi $\mathrm{Y}$, Matsuura N, Toshima H, Itoh N, Ishikawa J, Mikami $\mathrm{Y}$, et al. Cloning of the gene cluster responsible for the biosynthesis of brasilicardin $\mathrm{A}$, a unique diterpenoid. $\mathrm{J}$ Antibiot 2008;61:164 74 .

[19] Jiang Y, Wang H, Lu C, Ding Y, Li Y, Shen Y. Identification and characterization of the cuevaene $\mathrm{A}$ biosynthetic gene cluster in Streptomyces sp. LZ35. ChemBioChem 2013;14:1468 75.

[20] Rusnak F, Liu J, Quinn N, Berchtold GA, Walsh CT. Subcloning of the enterobactin biosynthetic gene entB: expression, purification, characterization and substrate specificity of isochorismatase. Biochemistry 1990;29:1425 35.

[21] Parsons JF, Calabrese K, Eisenstein E, Ladner JE. Structure and mechanism of Pseudomonas aeruginosa PhzD, an isochorismatase from the phenazine biosynthetic pathway. Biochemistry 2003;42:5684 93.

[22] Drake EJ, Nicolai DA, Gulick AM. Structure of the EntB multidomain nonribosomal peptide synthetase and functional analysis of its interaction with the EntE adenylation domain. Chem Biol 2006;13:409 19.

[23] Goral A, Tkaczuk K, Chruszcz M, Kagan O, Savchenko A, Minor W. Crystal structure of a putative isochorismatase hydrolase from Oleispira antarctica. J Struct Funct Genomics 2012;13:27 36.

[24] Knapik AA, Petkowski JJ, Otwinowski Z, Cymborowski MT, Cooper DR, Chruszcz M, et al. Structure of Escherichia coli RutC, a member of the YjgF family and putative aminoacrylate peracid reductase of the rut operon. Acta Crystallogr Sect F Struct Biol Cryst Commun 2012;68:1294 9.
[25] Sinha S, Rappu P, Lange SC, Mäntsälä P, Zalkin H, Smith JL. Crystal structure of Bacillus subtilis YabJ, a purine regulatory protein and member of the highly conserved YjgF family. Proc Natl Acad Sci USA 1999;96:13074 9.

[26] Thakur KG, Praveena T, Gopal B. Mycobacterium tuberculosis Rv2704 is a member of the YjgF/YER057c/UK114 family. Proteins 2010;78:773 8.

[27] Burman J, Stevenson C, Sawers RG, Lawson D. The crystal structure of Escherichia coli TdcF, a member of the highly conserved YjgF/YER057c/UK114 family. BMC Struct Biol 2007;7:30

[28] Lambrecht JA, Flynn JM, Downs DM. Conserved YjgF protein family deaminates reactive enamine/imine intermediates of pyridoxal 5'-phosphate (PLP)-dependent enzyme reactions. J Biol Chem 2012;287:3454 61.

[29] Ladner JE, Reddy P, Davis A, Tordova M, Howard AJ, Gilliland GL. The $1.30 \AA$ \& resolution structure of the Bacillus subtilis chorismate mutase catalytic homotrimer. Acta Crystallogr 2000;56:673 83.

[30] Hubrich F, Mordhorst S, Andexer JN. Cinnamic acid derivatives as inhibitors for chorismatases and isochorismatases. Bioorg Med Chem Lett 2013;23:1477 81.

[31] Holm L, Rosenström P. Dali server: conservation mapping in 3D. Nucleic Acids Res 2010;38:W545 9.

[32] Bornberg-Bauer E, Beaussart F, Kummerfeld SK, Teichmann SA, Weiner J. The evolution of domain arrangements in proteins and interaction networks. Cell Mol Life Sci 2005;62:435 45.

[33] Peat TS, Balotra S, Wilding M, French NG, Briggs LJ, Panjikar $S$, et al. Cyanuric acid hydrolase: evolutionary innovation by structural concatenation. Mol Microbiol 2013;88:1149 63.

[34] Kay BK, Williamson MP, Sudol M. The importance of being proline: the interaction of proline-rich motifs in signaling proteins with their cognate domains. FASEB $\mathrm{J}$ 2000;14:231 41.

[35] Guilford WJ, Copley SD, Knowles JR. The mechanism of the chorismate mutase reaction. J Am Chem Soc 1987;109:5013 9.

[36] Afshar C, Jaffe EK, Carrell HL, Markham GD, Rajagopalan JS, Rossi M, et al. The molecular conformation of chorismic acid in the crystalline state. Bioorg Chem 1992;20:323 33.

[37] Dias MVB, Huang F, Chirgadze DY, Tosin M, Spiteller D, Dry EFV, et al. Structural basis for the activity and substrate specificity of fluoroacetyl-CoA thioesterase FIK. J Biol Chem 2010;285:22495 504 .

[38] Kabsch W. Integration, scaling, space-group assignment and post-refinement. Acta Crystallogr 2010;66:133 44.

[39] Kabsch W. XDS. Acta Crystallogr 2010;66:125 32.

[40] Pape T, Schneider TR. HKL2MAP: a graphical user interface for macromolecular phasing with SHELX programs. J Appl Crystallogr 2004;37:843 4 .

[41] Emsley P, Cowtan K. Coot: model-building tools for molecular graphics. Acta Crystallogr 2004;60:2126 32.

[42] Afonine PV, Grosse-Kunstleve RW, Echols N, Headd JJ, Moriarty NW, Mustyakimov M, et al. Towards automated crystallographic structure refinement with phenix.refine. Acta Crystallogr 2012;68:352 67. 